\title{
Comparative Impact of Stirring and Shearing in Drug Dissolution Testing with USP Paddle and Crescent-Shaped Spindles
}

\author{
Saeed A. Qureshi \\ Banting Research Centre (A/L 2202C1), Tunney's Pasture, Ottawa, Ontario, K1A OL2 CANADA
}

\begin{abstract}
A new crescent-shaped spindle has been proposed to address artefacts found with the USP Paddle spindle. Because of improved stirring and mixing environment within dissolution vessels resulting in improved product-medium interactions, the crescent-shaped spindle provides a more appropriate dissolution test. Diltiazem immediate- (IR) and extended-release (ER) tablets and capsules were used as model drug products to demonstrate that the appropriate dissolution rate may be obtained using a single and lower spindle rotation speed of $25 \mathrm{rpm}$, rather than product-dependent higher rotation speeds of 75 and $100 \mathrm{rpm}$ with the USP Paddle. The inability to establish appropriate dissolution rate using single rpm for these two products using USP spindle appears to be because of its well documented artefacts of poor stirring and mixing environment in the vessels. There was no evidence of a crushing or harsh stirring environment using the crescent-shaped spindle, as compared to the USP Paddle spindle. This appears to confirm that increased dissolution rate using crescent-shaped spindle is because of the improved stirring environment. In addition, this study provides further evidence that products with different release characteristics (e.g., IR vs ER) may be analyzed using a single experimental condition (crescent-shaped spindle at 25 rpm) unlike the use of the USP Paddle spindle, which requires product dependent multiple test conditions. Thus, the use of the crescent-shaped spindle appears to provide a better alternative for improved dissolution testing and, in particular, for comparative purposes.
\end{abstract}

\section{Introduction}

D rug dissolution testing is an integral part of drug product development and quality assessment. Often drug dissolution testing is a regulatory requirement to establish the quality of a drug product [1-3].

In reality, drug dissolution testing is a solubility determination technique. It utilizes specialized equipment for that purpose, commonly known as basket and paddle apparatuses. These apparatuses have official recognition throughout the world, generally through pharmacopoeias, such as USP, where these apparatuses are also referred to as Apparatuses 1 and 2, respectively [1].

It has been well documented that results obtained from dissolution testing using these apparatuses may be highly variable and unpredictable [4-9] and often lack relevance to the in vivo drug release characteristics (bioavailability) of the products [10-11]. In recently reported studies [12-15], it has also been demonstrated that, because of the lack of an adequate stirring and mixing environment in the dissolution vessels, inaccurate comparative drug dissolution/release of the test products may also be observed.

To address these flaws and artefacts of the currently used apparatuses, we have proposed a new spindle for providing improved stirring and mixing and thus improved productmedium interaction [12-15]. This report provides further evidence in support of the superiority of the crescentshaped spindle over the currently used spindles.

\section{Experimental}

\section{Materials and Methods}

Pharmaceutical Products: IR (60-mg) tablet and ER (120$\mathrm{mg}$ ) capsule diltiazem products used in the study were obtained from the local Canadian market.
All other chemicals and solvents were of analytical grade and used as supplied by the suppliers.

Stirring Spindle: The crescent-shaped [14] and USP spindles were used for testing. The crescent-shaped spindle is designed to fit the commonly used apparatus as a substitute for the paddle or basket spindle. The agitator has a stem part, and the lower half is curved to conform to the shape of the vessel in which it is rotated, but with no direct contact with the surface of the vessel.

The lower component (curved brush) is shaped from a commercially available brush made of stiff twisted metal wires and fine bristles (Fisher Scientific, Cat. \# 03-578). Their use over the years has raised no quality control or variability issues and has shown excellent durability and reproducibility.

The end of the stem, conforming to the bottom part of the vessel, has filamentary elements (bristles) filling the gap between the stem and the bottom part of the vessel. It is important to note that unlike the use of the USP Paddle spindle where there is a gap $(25 \mathrm{~mm})$ between the paddle bottom and vessel surface, the crescent-shaped spindle does not have such a gap. The bristles always are in contact with the surface of the vessel. Therefore, when the device is attached to the vertical shaft and rotates, the brush-type agitator sweeps through the bottom and sides of the vessel accomplishing even distribution and mixing of the disintegrating material and also avoiding any accumulation (coning).

Instrumentation: The dissolution tests were conducted using a VanKel system (VK 700) comprising a bath with six vessels and meeting the physical and mechanical specifications as required by the USP [1]. The dissolution tests were conducted using the crescent-shaped spindle at $25 \mathrm{rpm}$ and 


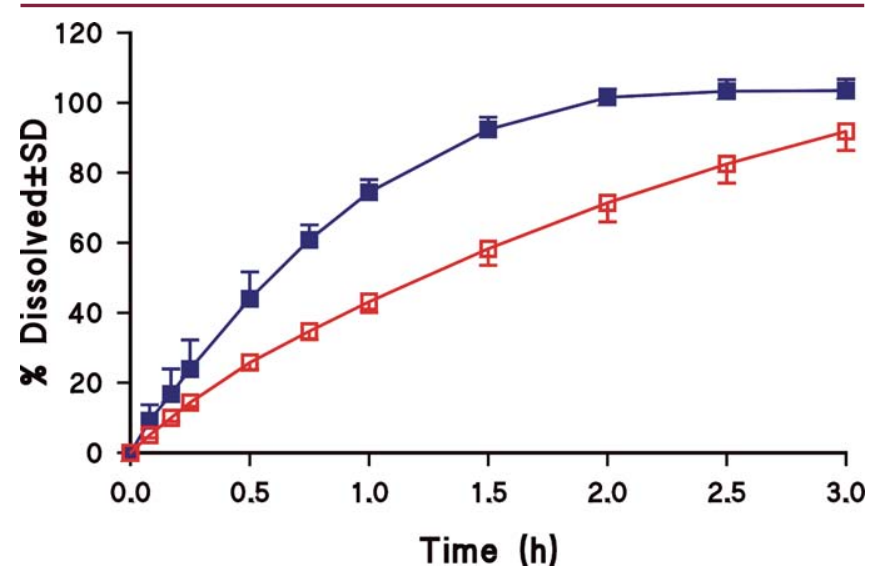

Figure 1: Drug release profiles of diltiazem $(60 \mathrm{mg})$ IR tablets $(n=6)$ with the crescent-shaped spindle at $25 \mathrm{rpm}$ (- - - ) and the USP Paddle spindle at $75 \mathrm{rpm}$ (-口-).

USP Paddle spindle at 75 (diltiazem IR tablets) and $100 \mathrm{rpm}$ (diltiazem ER capsules).

The tests for diltiazem products were conducted using $900 \mathrm{~mL}$ of water. The amount of diltiazem dissolved for each vessel was determined at various time intervals up to three hours for the IR product and 24 hours for the ER product, respectively. The quantitation was achieved by ultraviolet absorbance at $240 \mathrm{~nm}$ of filtered portions of the solution under test, in comparison with a reference solution having a known concentration of diltiazem standard [16].

Prior to use, the dissolution media were equilibrated at 37 ${ }^{\circ} \mathrm{C}$ overnight to deaerate the medium so that bubble formation due to escape of dissolved gases was minimized during the test.

Deaeration of the dissolution medium by equilibration: It is generally recommended that drug dissolution testing be conducted using deaerated dissolution medium. The medium is deaerated to avoid bubble formation during testing; bubbles may stick to the surfaces (vessel, spindle, and/or products) and may cause high variation in results. The air bubble formation may occur because of the solubility differentials of gasses (not only oxygen) in the medium at room temperature vs $37^{\circ} \mathrm{C}$, at which dissolution tests are conducted. During the medium transfer from room temperature to dissolution vessels and equilibration at 37 ${ }^{\circ} \mathrm{C}$, this solubility differential (higher gases solubility/concentration at room temperature than at $37^{\circ} \mathrm{C}$ ) causes the dissolved gasses to escape in the form of bubbles. To minimize bubble formation, it is usually recommended that medium be deaerated by degassing under low pressures (vacuum), with or without heating (often to $41^{\circ} \mathrm{C}$ ), or sparging with lower solubility gasses, such as helium.

Therefore, in reality, the bubble formation and its potential negative impact are the result of lack of allowance of suffi-
Table 1: Percent drug release values (mean; standard deviations, SD; and percent relative standard deviations, \%RSD) for 60-mg diltiazem IR tablet product using different spindles.

\begin{tabular}{|ccccc|}
\hline & \multicolumn{2}{c}{$\begin{array}{c}\text { USP Paddle } \\
\text { Spindle(75 rpm) }\end{array}$} & \multicolumn{2}{c|}{$\begin{array}{c}\text { Crescent-shaped } \\
\text { spindle (25 rpm) }\end{array}$} \\
\cline { 2 - 5 } $\begin{array}{c}\text { Sampling } \\
\text { Time (h) }\end{array}$ & $\begin{array}{c}\text { Mean } \\
(\mathrm{n}=6)\end{array}$ & $\begin{array}{c}\text { SD } \\
(\% \mathrm{RSD})\end{array}$ & $\begin{array}{c}\text { Mean } \\
(\mathrm{n}=6)\end{array}$ & $\begin{array}{c}\text { SD } \\
(\% \mathrm{RSD})\end{array}$ \\
\hline 0.08 & 5.1 & $0.8(16.1)$ & 9.2 & $4.5(49.5)$ \\
\hline 0.17 & 10.0 & $1.0(10.3)$ & 16.8 & $7.2(42.8)$ \\
\hline 0.25 & 14.4 & $1.2(8.1)$ & 23.9 & $8.3(34.7)$ \\
\hline 0.50 & 25.8 & $2.0(7.6)$ & 44.0 & $7.7(17.4)$ \\
\hline 0.75 & 34.7 & $2.4(6.9)$ & 60.8 & $4.4(7.1)$ \\
\hline 1.00 & 43.2 & $3.1(7.1)$ & 74.4 & $3.6(4.8)$ \\
\hline 1.50 & 58.3 & $4.7(8.1)$ & 92.4 & $3.5(3.8)$ \\
\hline 2.00 & 71.4 & $5.5(7.6)$ & 101.6 & $2.4(2.3)$ \\
\hline 2.50 & 82.5 & $5.4(6.6)$ & 103.3 & $3.3(3.2)$ \\
\hline 3.00 & 91.8 & $5.4(5.9)$ & 103.5 & $3.3(3.2)$ \\
\hline
\end{tabular}

cient time for equilibration of dissolution medium at $37{ }^{\circ} \mathrm{C}$. If this equilibration is achieved prior to the initiation of the dissolution test, then the system would be free from bubble formation and provide a stable experimental environment throughout the testing period. Therefore, equilibration at 37 ${ }^{\circ} \mathrm{C}$ for an extended period of time ( 24 hours) may be considered as a deaeration approach as it reduces the content of dissolved gasses in the medium and appears to provide improved dissolution testing [8].

Data Analysis: The data were collated and analysed using SAS software (SAS Institute, Cary, NC).

\section{Results}

Figure 1 shows drug release characteristics of IR diltiazem tablets using both spindles, rotating at 25 (crescent-shaped) and $75 \mathrm{rpm}$ (USP Paddle). The corresponding data along with calculated SD and \%RSD are tabulated in Table 1. As expected, drug release was faster with a crescent-shaped spindle because dissolution occurs from all sides of the tablet, while with a paddle spindle, no or limited dissolution occurs from the surface of the tablet that touches the surface of the vessel [17]. The dissolution maximum (plateau) is reached in about two hours using the crescent-shaped spindle, while with the USP Paddle, a plateau does not appear to be reached in three hours, indicating incomplete dissolution. The operational views of dissolution testing using both spindles are shown in Figure 2. The photographs were taken 

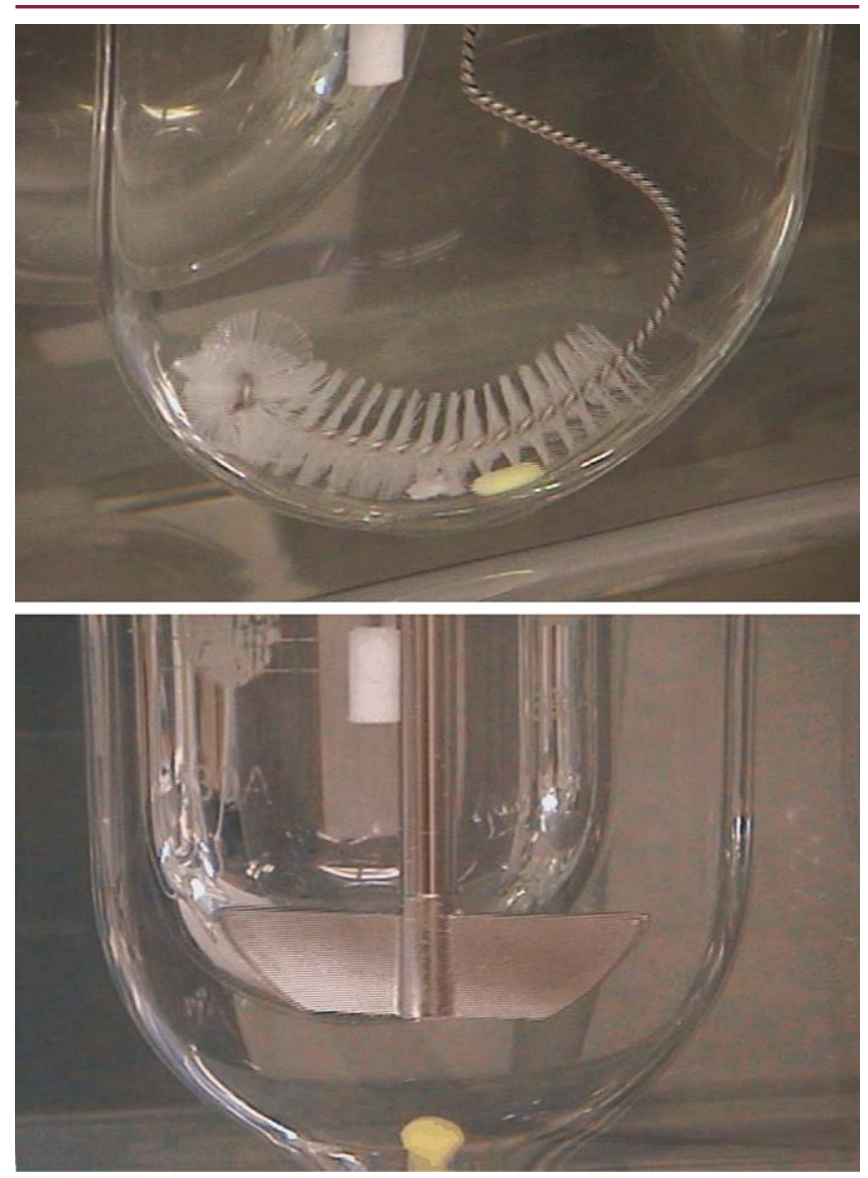

Figure 2: Photographic images of the diltiazem (60-mg) IR tablet behavior following 45 minutes of testing using the crescent-shaped spindle at $25 \mathrm{rpm}$ (above) and the USP Paddle spindle at $75 \mathrm{rpm}$ (below).

following 45 minutes of stirring using both spindles, clearly showing maintenance of tablet integrity.

Figures 3 shows drug release profiles of the ER diltiazem capsule product. The corresponding data along with calculated SD and \%RSD are tabulated in Table 2. The ER product shows bi-phasic dissolution characteristics using both the spindles, rotating at $25 \mathrm{rpm}$ (crescent-shaped) and $100 \mathrm{rpm}$ (USP Paddle). The physical behavior of the product (beads from the capsules shells) as observed after three hours of dissolution is shown in Figure 4. There were no apparent differences in the integrity of beads using either spindle. Drug dissolution characteristics up to 12 hours appear to be quite similar using either spindle; however, the amount dissolved at later times was significantly higher using the crescent-shaped spindle than with USP Paddle spindle. In both cases, the maximum (plateau) dissolution appears to be obtained at 21 hours indicating complete dissolution from the products. Plateau levels are slightly different with two spindles, i.e., lower with USP spindle than with the crescent-shaped spindle.

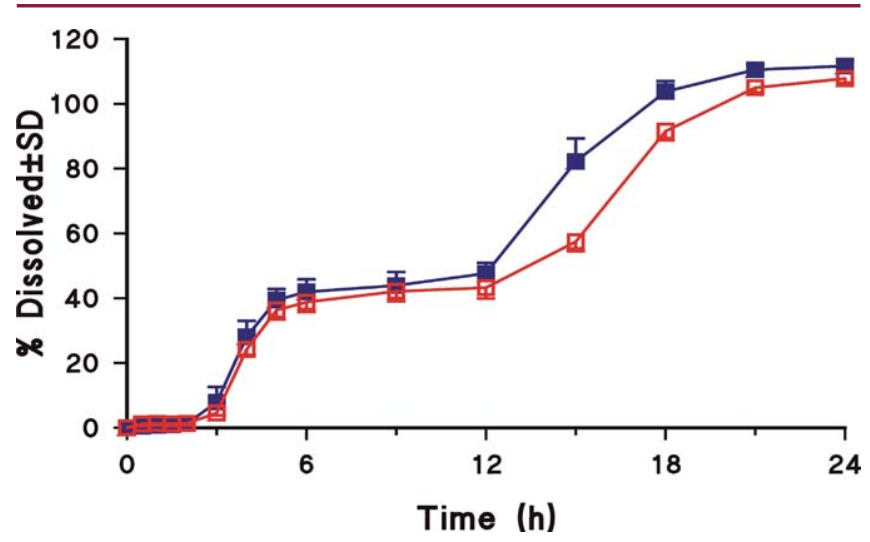

Figure 3: Drug release profiles of diltiazem (120 mg) ER capsules $(n=6)$ with the crescent-shaped spindle at $25 \mathrm{rpm}(-\square-)$ and the USP Paddle spindle at $100 \mathrm{rpm}$ (一 $\square-$ ).

\section{Discussion}

One of the commonly used apparatuses for dissolution testing is the USP Paddle Apparatus. It has been shown that this apparatus does not provide adequate stirring and mixing, resulting in pockets of unstirred area and variable hydrodynamics, thus giving rise to dissolution results that are usually highly variable and unpredictable $[4,8,9]$. In addition, product settling effects (positions of the tablets/capsules at the base of vessels) and no or minimal reach of the dissolution medium at the side of the product that touches the vessel surfaces exaggerate variability in rate and extent of dissolution. To address these artefacts of the USP Paddle apparatus, a new crescent-shaped spindle has been proposed [13-14].

The design of the crescent-shaped spindle/stirrer is such that it addresses the above mentioned artefacts of the USP apparatus. The new spindle, which rotates and sweeps or rakes through the bottom of the dissolution vessel, will thus alleviate problems associated with the unstirred zone (cone formation), positioning and settling effect of product at the bottom of vessel and will thus improve solute-solvent interaction from all sides of tablets/products. In addition, the spindle helps spread the disintegrated material, thus increasing surface area (as one would anticipate in vivo) for better dissolution. Thus, the use of the new spindle provides improved dissolution results and provides a more appropriate reflection of the in vivo physiological environment experienced by orally ingested product.

Based on the above mentioned artefacts, it has been clearly demonstrated that the USP Paddle spindle will inaccurately characterize the dissolution behavior of test products, particularly those which disintegrate and accumulate at the bottom of the vessel [13-15]. It has been demonstrated that, in such cases, slower and lower drug release is the result of the artefact of the technique. The crescent 
Table 2: Percent drug release values (mean; standard deviations, SD; and percent relative standard deviations, \%RSD) for 120-mg diltiazem ER capsule product using different spindles.

\begin{tabular}{|c|c|c|c|c|}
\hline \multirow[b]{2}{*}{$\begin{array}{l}\text { Sampling } \\
\text { Time (h) }\end{array}$} & \multicolumn{2}{|c|}{$\begin{array}{l}\text { USP Paddle Spindle } \\
\text { (100 rpm) }\end{array}$} & \multicolumn{2}{|c|}{$\begin{array}{l}\text { Crescent-shaped } \\
\text { spindle (25 rpm) }\end{array}$} \\
\hline & $\begin{array}{l}\text { Mean } \\
(\mathrm{n}=6)\end{array}$ & $\begin{array}{c}\text { SD } \\
(\% \mathrm{RSD})\end{array}$ & $\begin{array}{l}\text { Mean } \\
(n=6)\end{array}$ & $\begin{array}{c}\text { SD } \\
(\% \text { RSD) }\end{array}$ \\
\hline 0.5 & 1.2 & $0.5(43.2)$ & 0.7 & $0.8(112.5)$ \\
\hline 1.0 & 1.3 & $0.5(39.9)$ & 0.9 & $0.8(91.5)$ \\
\hline 1.5 & 1.2 & $0.6(48.2)$ & 1.0 & $0.9(83.8)$ \\
\hline 2.0 & 1.4 & $0.5(39.7)$ & 1.3 & $0.9(66.6)$ \\
\hline 3.0 & 4.6 & $0.8(18.0)$ & 8.0 & $4.7(58.7)$ \\
\hline 4.0 & 24.2 & $1.7(7.2)$ & 28.1 & $4.9(17.3)$ \\
\hline 5.0 & 36.3 & $2.9(8.0)$ & 39.4 & $3.4(8.6)$ \\
\hline 6.0 & 38.8 & $3.0(7.7)$ & 42.0 & $3.9(9.3)$ \\
\hline 9.0 & 42.1 & $3.1(7.3)$ & 43.9 & $4.2(9.5)$ \\
\hline 12.0 & 43.2 & 3.3 (7.6) & 47.6 & $3.3(6.9)$ \\
\hline 15.0 & 57.5 & $2.9(5.1)$ & 82.2 & $7.1(8.7)$ \\
\hline 18.0 & 91.5 & $2.8(3.0)$ & 103.8 & $3.2(3.1)$ \\
\hline 21.0 & 105.0 & $1.4(1.3)$ & 110.5 & $1.6(1.4)$ \\
\hline 24.0 & 107.8 & $1.3(1.2)$ & 111.7 & $1.6(1.5)$ \\
\hline
\end{tabular}

shape does not allow cone formation and assists in spreading the disintegrated material, resulting in increased surface area and thus better interaction of solute with medium, providing a higher and more accurate dissolution rate [15]. However, concerns have been expressed that higher dissolution rates using the crescent-shaped spindle may be due to the higher shearing impact, and its effect may be compared to that of the use of a Waring blender [18].

This study was performed to demonstrate that the shearing impact of the crescent-shaped spindle is the same as or similar to that of USP Paddle. The two products tested were diltiazem IR tablet and ER capsule. In both cases, the drug release is formulation controlled, i.e., products do not disintegrate and release the drug instantaneously. Even drug release from IR tablets is expected to take three hours for complete dissolution as per USP monograph requirements [16]. For the ER capsules, the required testing time is 24 hours. Therefore, any crushing or harsh stirring effects of the crescent-shaped spindle would result in significantly faster, and possibly abrupt, drug release rather than the expected gradual release.

As is demonstrated in Figure 2, following 45 minutes of
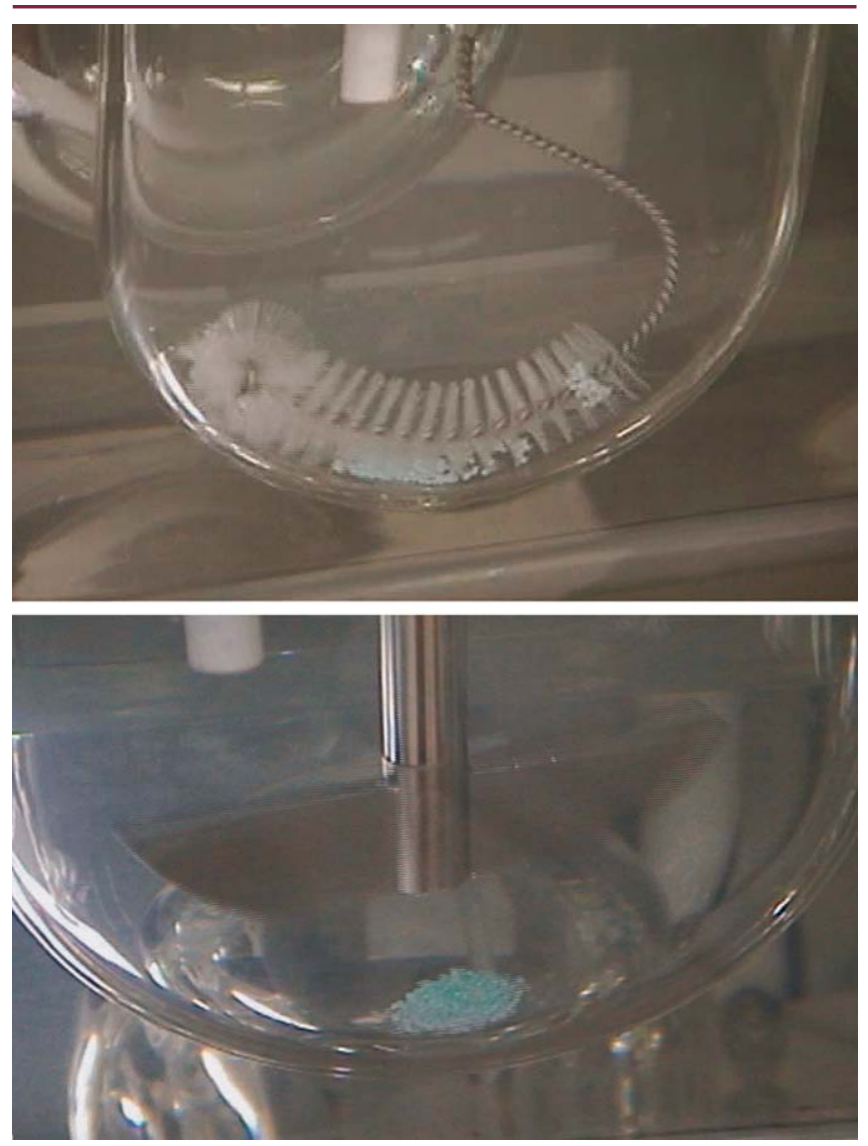

Figure 4: Photographic images of the diltiazem (120-mg) ER capsules (beads) behavior following three hours of testing using the crescent-shaped spindle at $25 \mathrm{rpm}$ (above) and the USP Paddle spindle at $100 \mathrm{rpm}$ (below).

dissolution testing, the physical state of the tablets with the two spindles is similar; in both cases the integrity of tablets remains intact. The faster or higher dissolution (Figure 1) using the crescent-shaped spindle appears to be due to improved interaction of tablets with dissolution medium as tablets move with the spindle. Also, tablets are usually settled somewhat off center, which is considered a better stirred area than the center, which also helps in improving dissolution. On the other hand, in the USP Paddle Apparatus, settling of tablets at the base of the vessels (center), which is well established as a least stirred zone, causes slower and lower dissolution [5,9]. Therefore, it may be concluded that the shearing impact of the crescent-shaped spindle is similar to that of the paddle, but due to improved interaction of product-medium, the crescent-shaped spindle, even at lower rotation speed of $25 \mathrm{rpm}$ compared to $75 \mathrm{rpm}$ for paddle, provides an efficient stirring effect and thus better dissolution results.

Figure 4 shows drug dissolution behavior using the crescent-shaped and USP Paddle spindles for the capsule product (beads) after 3 hours of dissolution. Beads 
remained intact and more spread out with the constant stirring and mixing of the crescent-shaped spindle, but formed a mound ("cone") with the USP Paddle spindle, even at 100 $\mathrm{rpm}$. Note that with both spindles, there was minimal release of the drug during the first three hours, establishing that there is no harsh or excessive crushing or rubbing behavior of the crescent-shaped spindle. Therefore, this study provides experimental evidence that, while addressing lack of stirring and mixing artefacts of the USP Paddle spindle, the crescent-shaped spindle creates an environment no harsher than that from the USP Paddle spindle. Drug dissolution results using the crescent-shaped stirrer are similar to those with the USP Paddle up to at least 12 hours. However, after 12 hours, dissolution was significantly faster with the crescent-shaped spindle than with the paddle. The reason for this behavior is not clear at this time. However, we would speculate that after soaking of the beads in the medium for 12 hours, the coating has been "softened," and with a better stirring and mixing environment using the crescent-shaped spindle, drug appears to dissolve faster as in the case of IR tablets. However, as such a stirring and mixing environment is lacking with the USP Paddle, the dissolution took more time (as with tablets using Paddle) reflecting the deficiency of the USP Paddle spindle rather than the release characteristics of the product.

With regard to within run (between units) variability aspect, a closer look at the data presented in the Tables 1 and 2 provides an interesting pattern of variability components obtained by using the two type of spindles. For the IR product, at earlier sampling times, i.e., up to half an hour, variability was higher using the crescent-shaped spindle. However, at the later times, variability using the crescentshaped spindle was significantly lower than with the paddle method. In case of the ER product, if one ignores the results and associated variability up to two hours, where product remained undissolved and it may be assumed that observed results are experimental error, variability in results from both spindles appears similar except for three sampling times, i.e., at 3,4 and 15 hours. At these three sampling times, there are sharp increases in dissolution rate, apparently from softening and/or peeling of the surfaces of the beads. Like in the IR tablet case where the earlier phase provides higher variability with the crescent-shaped spindle, similar higher variabilities are observed with ER products during these so-called earlier phases. It is highly likely that these are reflective of product-dependant characteristics such as disintegration, softening, and peeling of the tablets/bead surfaces and are pronounced with the use of crescent-shaped spindle, which provides efficient product-medium interaction.

Generally it is presumed that the crescent shape may provide harsher and stronger agitation that causes it to provide higher dissolution rate and probably less variable results [18]. This study does not appear to support this assumption. In fact, in some cases, the crescent-shaped spindle may provide higher unit-to-unit variability than the USP Paddle spindle, presumably better reflecting product characteristics. Thus, the crescent-shaped spindle not only provides more efficient stirring and mixing, resulting in improved dissolution rate, but also better unit-to-unit discrimination.

In addition to improved drug dissolution testing, use of the crescent-shaped spindle provides two other distinct advantages over the use USP Paddle spindle and possibly the basket;

1. Products of different pharmaceutical attributes (e.g., release types, IR vs ER) can be analysed using a single set of experimental conditions, thus providing appropriate comparative dissolution testing. The use of the USP Paddle spindle requires two or more product-dependent experimental conditions, and thus comparison of dissolution/release characteristics between products, such as in this case of IR and ER diltiazem products, may not be possible.

From a physiological perspective, testing using a single set of experimental conditions is also desirable as both IR and ER products are developed for the same physiological environment. Therefore, science dictates that an in vitro test should also be conducted using a single testing environment. The use of the crescent-shaped spindle provides such a desired option.

2. At the end of the testing using the crescent-shaped spindle, a distinct plateau phase is usually observed at $100 \%$ drug release, indicating that complete release/dissolution has been achieved. However, the plateau phase using the USP Paddle spindle sometimes is not achieved, as appears to be case for the IR diltiazem tablets. The percent-release values at the last sampling time were in the range of $86-100$. It is possible that by extending the testing time, these values will further increase or, as often happens, that a plateau is reached at lower than 100\% with the USP Paddle. The reason for reaching a plateau of less than $100 \%$ is that often a small portion of the drug resides in the dead/unstirred zone at the bottom of the vessel. Therefore, even though products are usually capable of releasing $100 \%$ of the drug, because of the artefact of unstirred zone, tolerances are usually set lower than $100 \%$, commonly at $80 \%$ or less. Therefore, it will not be possible to assess whether lower drug release is product related or due to the artefact of this dead zone in the vessel. As the use of the crescentshaped spindle avoids formation of a dead zone, one should expect a plateau of $100 \%$ drug release. Absence of that should indicate product (potency) related issues. 
Therefore, dissolution testing using the crescent-shaped spindle not only provides improved testing but also provides a more accurate comparative evaluation of products as well as a better reflection of total releasable drug content of the product.

In conclusion, it can be stated that the crescent-shaped spindle at $25 \mathrm{rpm}$ provides better stirring and mixing but similar shearing impact to that of the paddle at 75 or 100 $\mathrm{rpm}$. It thus yields improved dissolution results and should be considered as a better alternate to the use of the USP Paddle and possibly the Basket spindles.

\section{Acknowledgment}

The skillful technical help provided by Dr. J. Dapaah is greatly appreciated.

\section{References}

1. General Chapters $\langle 711\rangle$ and $\langle 724\rangle$, United States Pharmacopeia 28, The United States Pharmacopeial Convention, Inc.: Rockville, MD, 2005, pp 2412-2422.

2. Guidance for Industry: Dissolution Testing of Immediate Release Solid Oral Dosage Forms. U.S. Department of Health and Human Services, Food and Drug Administration, U.S. Government Printing Office: Washington, DC, 1997, http://www.fda.gov/cder/ guidance/1713bp1.pdf.

3. Guidance for Industry: Extended Release Oral Dosage Forms: Development, Evaluation, and Application of In Vitro/In Vivo Correlations. U.S. Department of Health and Human Services, Food and Drug Administration, U.S. Government Printing Office:Washington, DC, 1997, http://www.fda.gov/cder/guidance/1306fnl.pdf.

4. McCarthy, L. G.; Kosiol, C.; Healy, A. M.; Bradley, G.; Sexton, J. C.; Corngan, O. I. Simulating the Hydrodynamic Conditions in the United States Pharmacopeia Paddle Dissolution Apparatus. AAPS PharmSciTech 2003, 4, 172-187.

5. McCarthy, L. G.; Bradley, G.; Sexton, J. C.; Corrigan, O. I.; Healy, A. M. Computational Fluid Dynamics Modeling of the Paddle Dissolution Apparatus: Agitation Rate, Mixing Patterns, and Fluid Velocities. AAPS PharmSciTech. 2004, 5, 1-10.

6. Qureshi, S. A.; McGilveray, I. J. A Critical Assessment of the USP Dissolution Apparatus Suitability Test Criteria. Drug Develop. Ind. Pharm. 1995, 21, 905-992.

7. Qureshi, S. A.; McGilveray, I. J. Assessment of Pharmaceutical Quality of Furosemide Tablets From Multinational Markets. Drug Devel. Ind. Pharm. 1998, 24, 995-1005.

8. Qureshi, S. A.; McGilveray, I. J. Typical Variability in Drug Dissolution Testing: Study with USP and FDA Calibrator Tablets and a Marketed Drug (Glibenclamide) Product. Eur.J. Pharm. Sci. 1999, 7, 249-258.

9. Muzzio, F. J.; Kukura, J.; Baxter, J. L. Hydrodynamicsinduced Variability in the USP II Dissolution Test. Int.J. Pharm. 2005, 292, 17-28.

10. Meyer, M. C.; Straughn, A. B.; Mhatre, R. M.;Shah, V. P.; Williams, R. L.; Lesko, L. J. Lack of In Vivo/In Vitro Correlations for $50 \mathrm{mg}$ and $250 \mathrm{mg}$ Primidone Tablets. Pharm. Res. 1998, 15, 1085-1089.

11. Repta, A. J. Dissolution Specifications for Drug Products. AAPS Pharmsci. 1999, Suppl., 2531.

12. Qureshi, S. A. Choice of Rotation Speed (rpm) for Biorelevant Drug Dissolution Testing Using a Crescentshape Spindle. Eur. J. Pharm. Sci. 2004, 23, 271-275.

13. Qureshi, S. A. Improved Drug Dissolution and Product Characterization Using the Crescent-shaped Spindle. J. Pharm. Pharmacol. 2004, 56, 1135-1141.

14. Qureshi, S. A.; Shabnam, J. Applications of a New Device (Spindle) for Improved Characterization of Drug Release (Dissolution) of Pharmaceutical Products. Eur.J. Pharm. Sci. 2003, 19, 291-297.

15. Qureshi, S.A. A New Crescent-shaped Spindle for Drug Dissolution Testing — but Why a New Spindle? Dissolution Technologies 2004, 11, 13-18.

16. Monograph: Diltiazem Hydrochloride Tablets, United States Pharmacopeia 28; The United States Pharmacopeial Convention, Inc.: Rockville, MD, 2005; pp 655-658.

17. Morihara, M.; Aoyagi, N.; Kaniwa, N.; Katori, N.; Kojim, S. Hydrodynamic Flows Around Tablets in Different Pharmacopeial Dissolution Tests. Drug Develop. Ind. Pharm. 2002, 28, 655-662.

18. Leeson, L.J.; Mayersohn, M. Letter to the Editor. Dissolution Technologies 2005, 12, 24-33. 